\section{SEM Provides Critical Process Information in Pharmaceutical Applications}

\author{
Ben Lich \\ FEI Company, Hillsboro, OR \\ (ben.lich@fei.com)
}

A number of trends in pharmaceutical manufacturing are driving rapid growth in the use of scanning electron microscopes (SEM) for high-resolution imaging and analysis. At the same time, continued development of SEM technology is enhancing system performance while simultaneously reducing usability barriers that have historically limited the acceptance of these techniques in pharmaceutical applications.

Among the most significant industry trends is a concerted effort by governmental regulatory agencies to encourage improvements in pharmaceutical manufacturing processes by applying the same scientific principles and engineering techniques that have lead to successful improvements in other industries, notably automobiles and semiconductors. The exceptionally high requirements for product quality within the pharmaceutical industry have generated a regulatory regime that imposes prohibitively high costs in both time and money to gain approval for process changes, even though the changes result in substantial performance gains. In the US, the Food and Drug Administration's Process Analytical Technology (PAT) initiative is intended to alleviate some of the approval burden by creating a regulatory framework that is more receptive to changes based on detailed knowledge and improved understanding of the manufacturing process. As the initiative's name implies, the anticipated improvements will rely heavily on better process analytical technologies, including SEM.

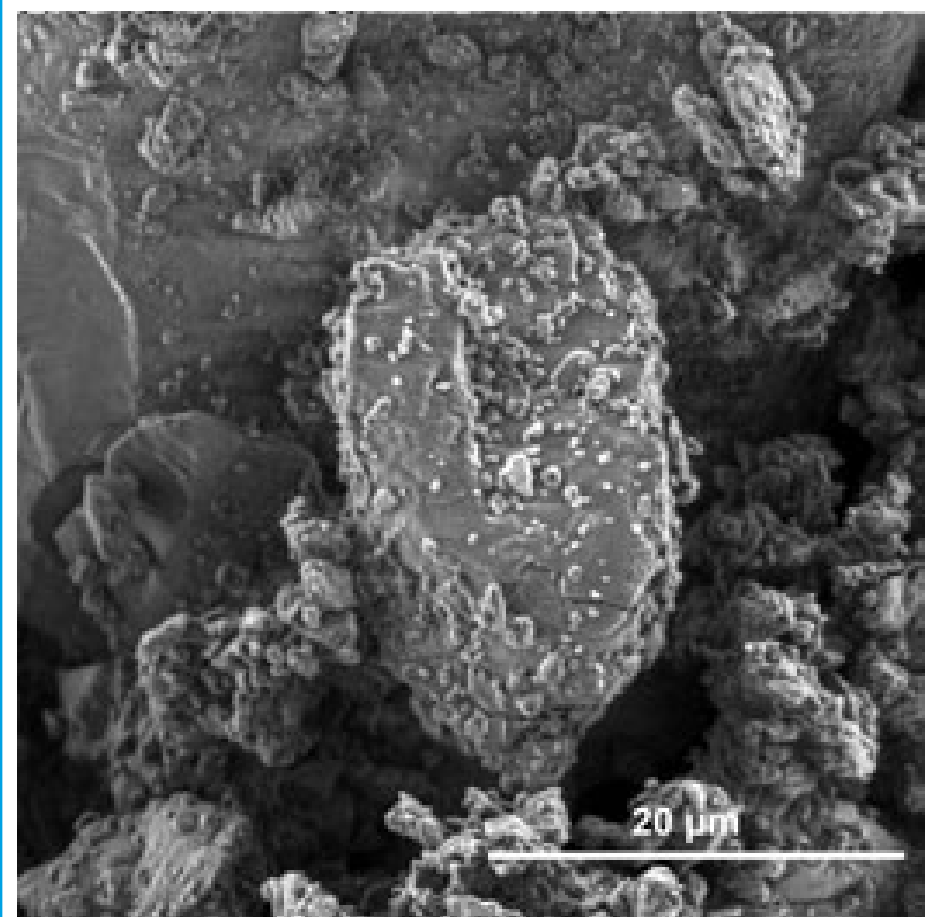

Figure 1: Image of formulated particles for inhalation used in a drypowder inhaler are shown here. The resolution reveals the small active compound particles on the larger carrier particles.
A second trend driving the demand for SEM is $t$ CrossMark ing size domain of critical features, such as particle sizes of active pharmaceutical ingredients (API). Somewhat ironically, this reduction in particle size is a result of an increase in the size and complexity of drug molecules. Larger molecules tend to be less soluble. Reducing the particle size increases surface area relative to volume, maintaining comparable overall solubility and bioavailability. Particle sizes in the deep sub micrometer to nanometer
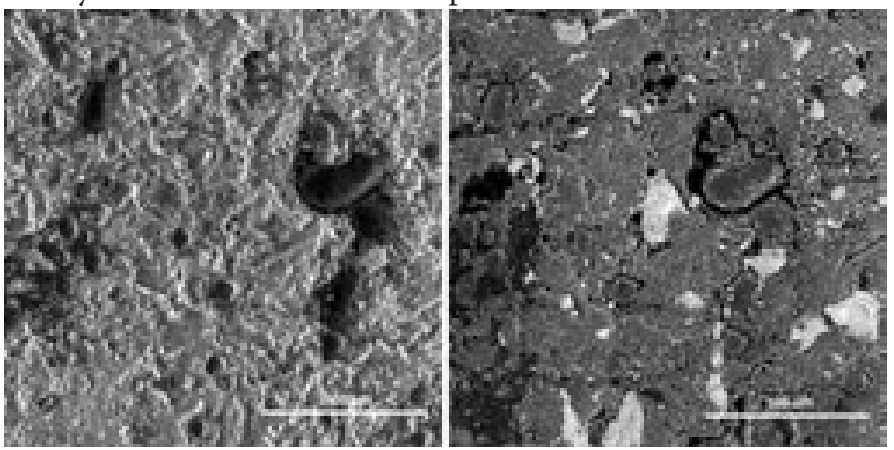

Figure 2 Left: this is a formulated paracetamol sample with enhanced imaging of the surface details with secondary electron information. Right: this is the same sample shown in the left image but with backscatter electron image information showing the material contrast. Note that the heavier elements appear brighter in the image.

ranges are not uncommon and clearly require the resolving power of an SEM for detailed morphological characterization.

The third trend contributing to the growth of SEM analysis is the dramatic improvements in microscope technology, especially with regard to usability. The latest generation SEM systems have made impressive advances in addressing the operational difficulty and sample constraints that limited the acceptance of their predecessors. Table top SEMs, such as the Phenom (FEI), now offer usable magnification up to 20,000X, twenty times better than typical optical microscopes; and they are arguably easier to use, with touch screen control and no sample preparation requirements. Even large, research grade instruments have been made easier to use with the addition of extensive, intelligent automation and greatly extended vacuum ranges that eliminate most sample constraints and preparation requirements. Environmental SEM (ESEM) can now operate with chamber pressures as high as 4000 $\mathrm{Pa}$ ( $\sim \%$ of sea level atmospheric pressure), sufficient to maintain a saturated water vapor environment at room temperature.

The new table-top SEMs may well find their way onto the production floor because of their low cost and ease of use. Larger, more powerful SEMs will likely remain in methods development organizations and quality control laboratories, where they will be used for initial validation during process development and the interpretation of anomalous results from production measurements made by other means. Fast, accurate imaging and analysis will shorten development and validation cycles and bring new products to market sooner. Likewise, in process and quality control applications, it will permit intuitive visual interpretation of batch-to-batch variations, resulting in fast correction of production problems and higher process yields.

\section{Surface characterization}

Surface characteristics can have significant impact on the behavior of drug formulations (figure 1). Accurate characterization requires detailed, unobscured observations of surface form 

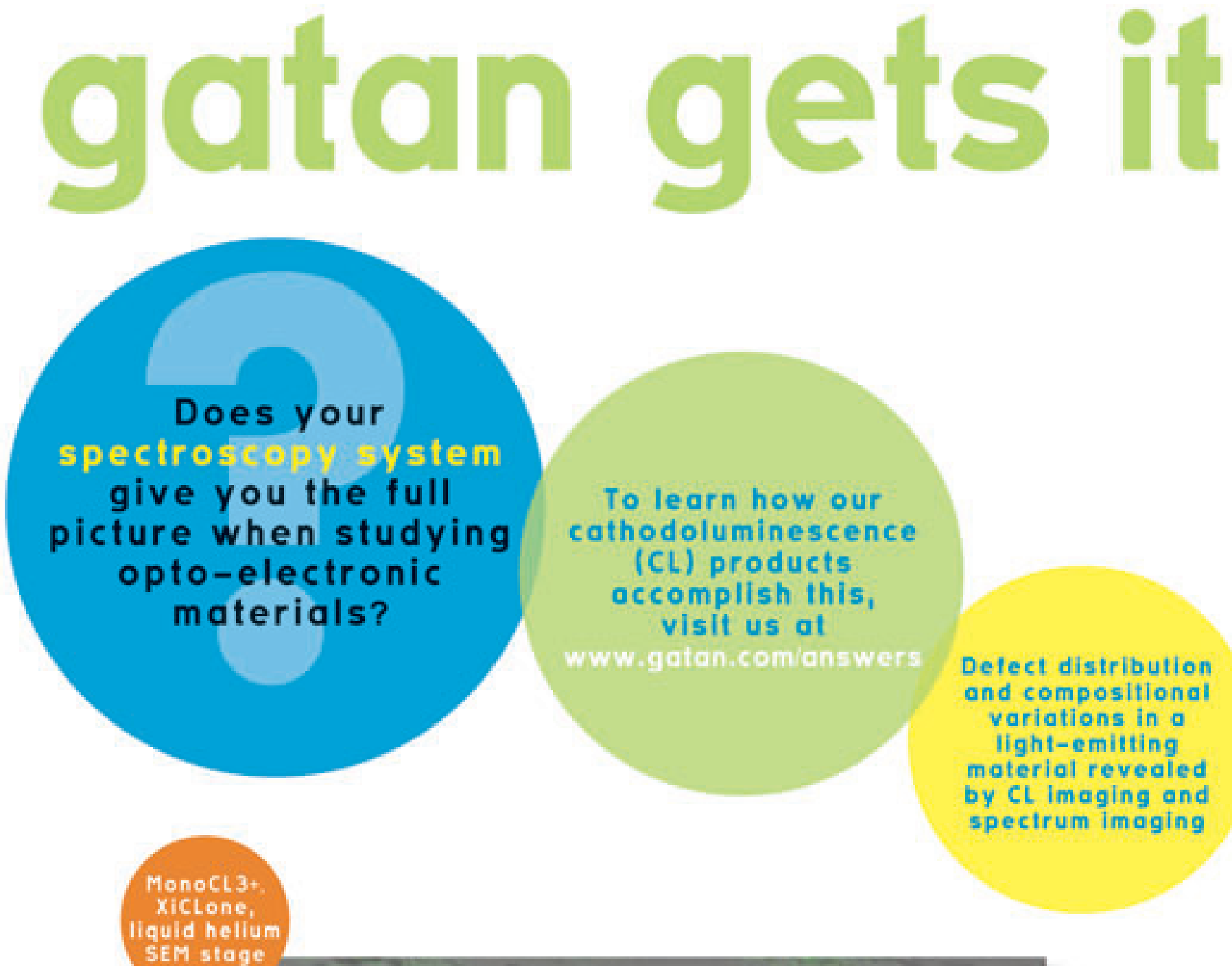

Defect distribution and compositional variations in a light-emitting material revealed by $\mathrm{CL}$ imaging and spectrum imaging

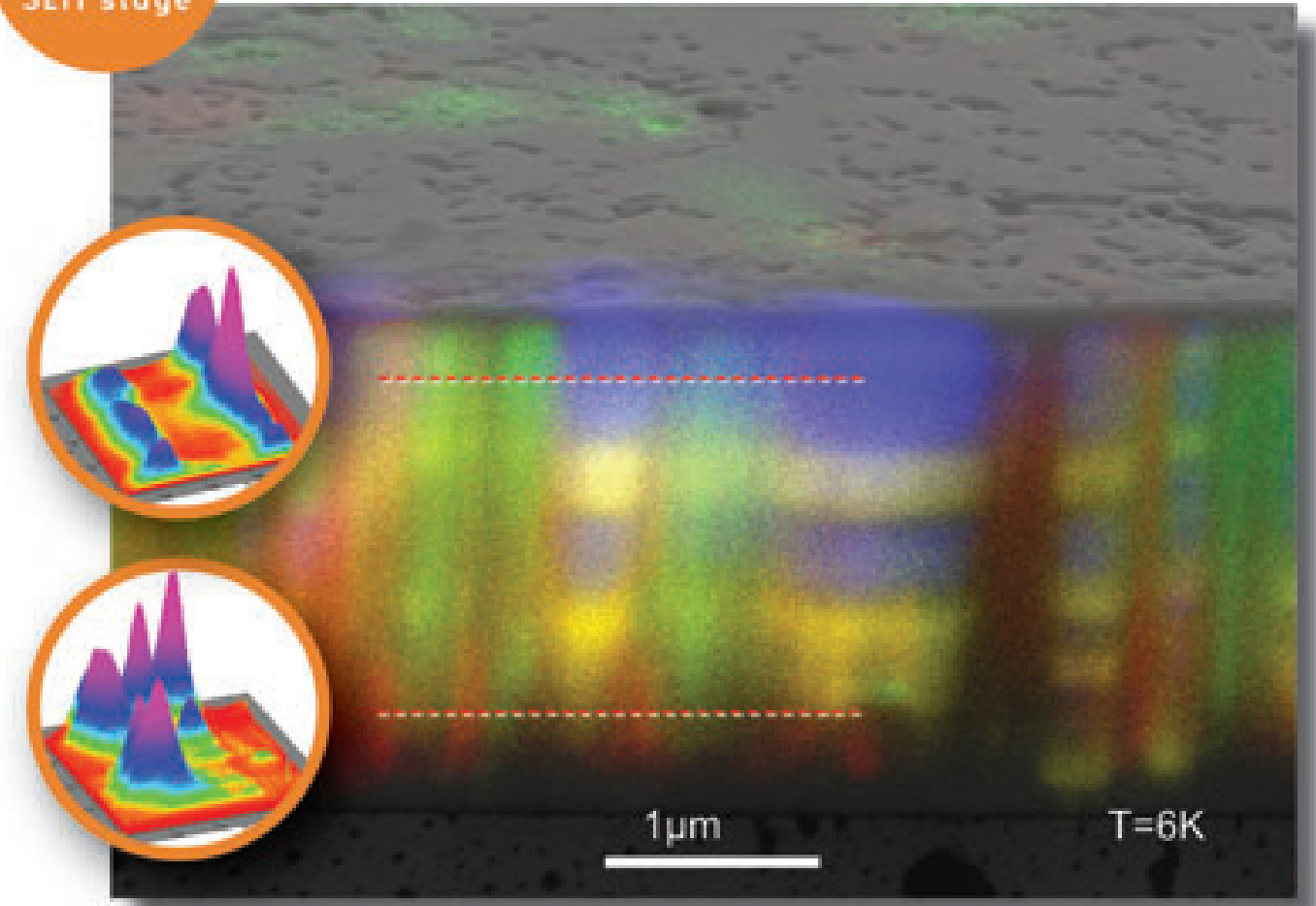

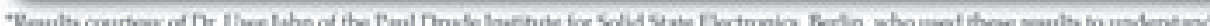

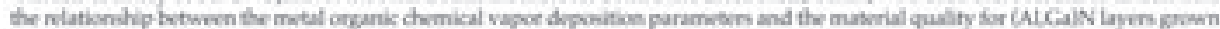

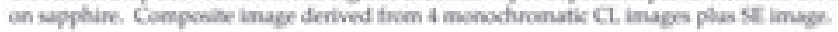




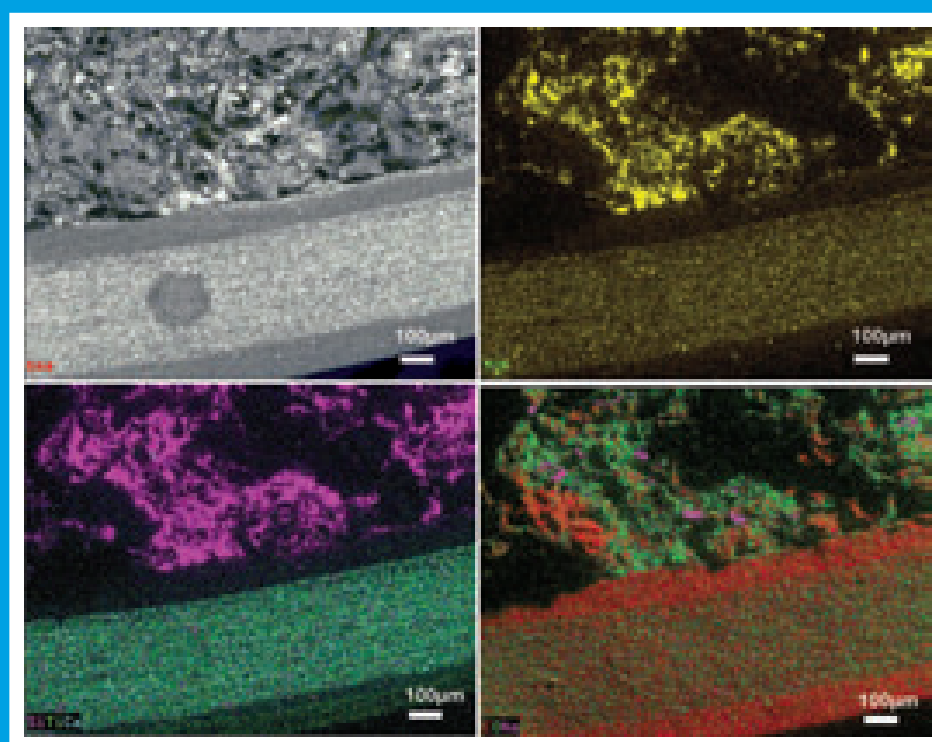

Figure 3 Far left - backscatter electron image of a formulated ibuprofen sample showing a part of the core, as well as the coating. Remaining images show pseudo colored elemental distribution: middle left-Mg (yellow), middle right $\mathrm{Si}$ (purple), Ti (green), Ca (blue); far right C (red), O (green), $\mathrm{Na}$ (purple).

and structure. The wide variety of signals generated in the SEM imaging process permits the selection of an imaging mode that best fits the analytical task at hand. Secondary electrons (SE) provide high resolution and emphasize topography. Backscattered electrons (BSE) bring out material contrast (figure 2). Characteristic X-rays show the location of specific elements (figure 3). Low voltage operation enhances near surface information. Wide field images permit rapid surveys of large areas.

Perhaps the most useful addition to conventional SEM for pharmaceutical manufacturing applications is the collection of technologies known as ESEM that permits imaging and analysis with very low vacuum (higher pressure) levels in the sample chamber. The ESEM detector actually uses gas in the sample chamber as an amplification medium for the secondary electron signal. Secondary electrons that escape from the sample surface are accelerated by an imposed detector field. They collide with and ionize gas molecules, thus creating additional electrons. The

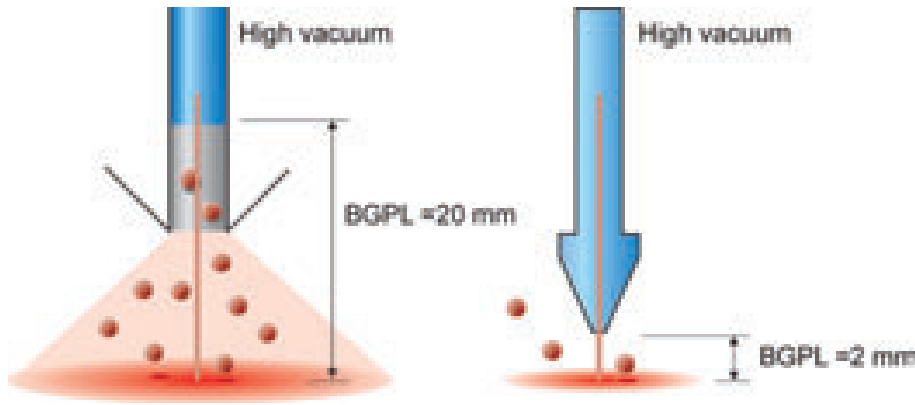

Figure 4 Left: typical low vacuum analytical geometry with long beam gas path length (BGPL) and large electron skirt. Right: proprietary ESEM analytical geometry with short BGPL.

process repeats itself, resulting in proportional, low noise, cascade amplification of the original secondary electron signal. In practical terms, ESEM enables immediate imaging of the non-conductive material without the conductive coatings required to prevent charge accumulation in high vacuum systems. In the ESEM, any charge accumulating at the sample surface is neutralized by the ions created by the amplification process.

\section{Blending homogeneity}

Blending is a critical step in many drug manufacturing processes. It is essential to understand how well the ingredients of the formulation are mixed, and to analyze the distribution of the active ingredients within the excipient matrix in the final product. For those formulations that allow characterization based on elemental information, the combination of secondary electron imaging, backscattered electron imaging and $\mathrm{x}$-ray analysis permits such an evaluation in a single tool (figure 3 ).

ESEM technology improves the accuracy of compositional analysis by eliminating potential interference caused by X-ray signals from conductive coatings. However, X-ray analysis in low vacuum conditions can be complicated by the scattering of beam electrons after they pass through the final pressure limiting aperture that separates the relatively high vacuum of the electron column from the gaseous environment of the sample chamber. In conventional approaches to low vacuum operation, the final optical aperture must also serve as the final pressure limiting aperture, forcing a compromise between electron optical imaging performance and X-ray analytical performance (Figure 4). The best position for the optical aperture is within the final lens, some distance from the sample surface. However, the longer the path between the aperture and the sample, the greater the chance a beam electron will be scattered by an encounter with a gas molecule, and the farther it is likely to land from the beam spot, where it can generate $\mathrm{x}$-rays that are not characteristic of the presumed beam location (Figure 5). ESEM solves this dilemma by using separate apertures for the optical and pressure limiting

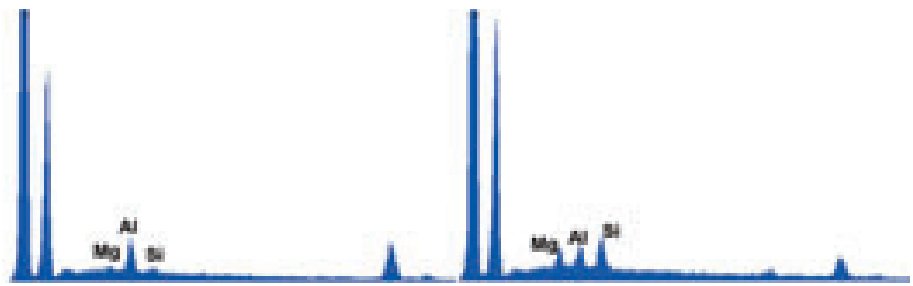

Figure 5 Two graphs showing the improved accuracy in energy dispersive spectroscopy (EDS) under low vacuum conditions. The graph on the left shows spurious EDS peaks from the surrounding region in a typical low vacuum EDS detection system. In the graph on the right, acquired in an ESEM with a shortened Beam Gas Path Length, the intensity of the spurious peaks is greatly reduced.

functions. The final optical aperture is optimally placed within the lens. The final pressure limiting aperture can be located close to the sample surface where it reduces the size and intensity of the 'skirt' created by scattered electrons, improving both the accuracy and spatial resolution of the $\mathrm{x}$-ray analysis.

\section{Raw materials: particle size, shape, and aggregation}

Particle size, shape and aggregation are primary determinants of dissolution behavior and drug bioavailability. They can also affect the flow characteristics of materials within the production process. Each characteristic can affect performance independently and requires independent control. Bulk techniques such as light scattering, are useful for detecting changes in these characteristics in a production environment but they generally cannot provide the information required to determine the detailed nature and cause of the change. Light microscopy can provide the needed morphological information but the sub micrometer and even nanometer size particles used in many modern formulations are 

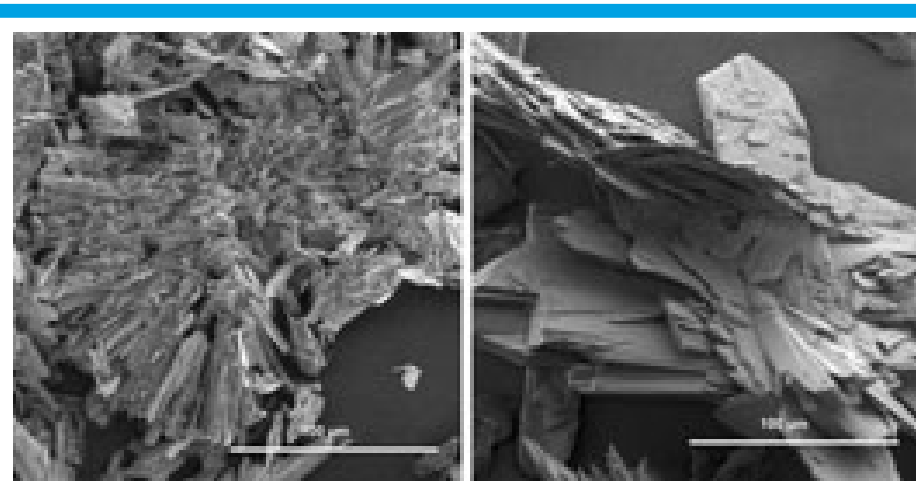

Figure 6 An active compound drug crystal after two supposedly identical crystallization processes. The images show the differences in the shape and size of the crystals.

well beyond their resolving power. High resolution SEM imaging provides direct visualization of size, shape and aggregation, permitting rapid assessment of process excursions and their root causes. Tighter control of these characteristics ensures predictable dissolution and bioavailability rates for drug compounds. SEM has the resolution needed for even the smallest particles and the dynamic range and depth of focus needed to image large particles as well.

Particle characterization can involve observations of particles from dry powders or from liquid suspensions. Typically, a liquid suspension is diluted and filtered to obtain a sample that can be analyzed in an SEM. Dry powders can be dispersed directly on a sample holder. For a conventional SEM, the particles, as well as
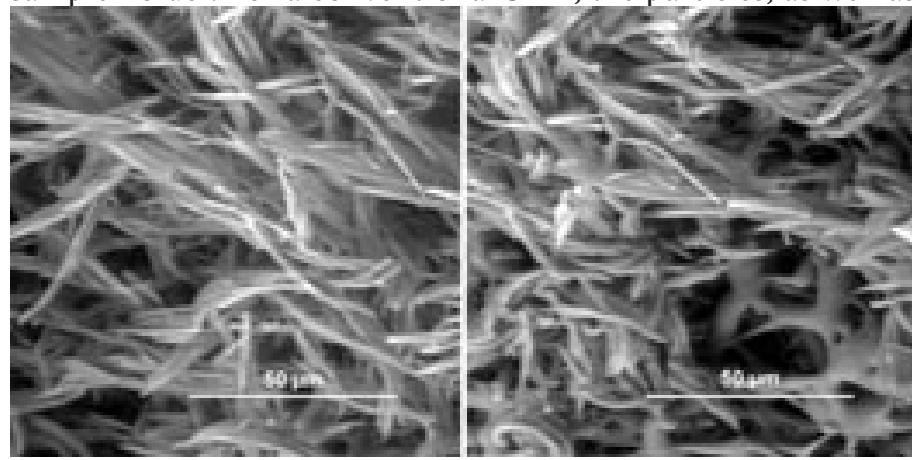

Figure 7 Direct visualization of Phase change effects of temperature on active compound crystals - left $81^{\circ} \mathrm{C}$, right $89^{\circ} \mathrm{C}$.

the filtration medium, must be dried and coated before analysis, potentially obscuring fine surface details that can be important for understanding milling and mixing processes. ESEM technology provides the ability to analyze size, shape and aggregation without coating, or even drying the filter before introducing it into the sample chamber.

\section{Dynamic experiments: Shelf life analysis}

A key issue in pharmaceutical formulation development is understanding how the product behaves in various ambient conditions. ESEM enables a fast, intuitive understanding of product behavior based on morphological changes that occur in response to variations in temperature or relative humidity. High temperature heating stages allow direct observations at temperatures up to $1000^{\circ} \mathrm{C}$. Peltier based stages can vary temperature \pm $30^{\circ} \mathrm{C}$ from ambient, and permit dynamic hydration/dehydration experiments as liquid condenses or evaporates at the sample. The latest generation of ESEM technology permits sample chamber pressures up to 4,000 $\mathrm{Pa}$ in the sample chamber. With this range
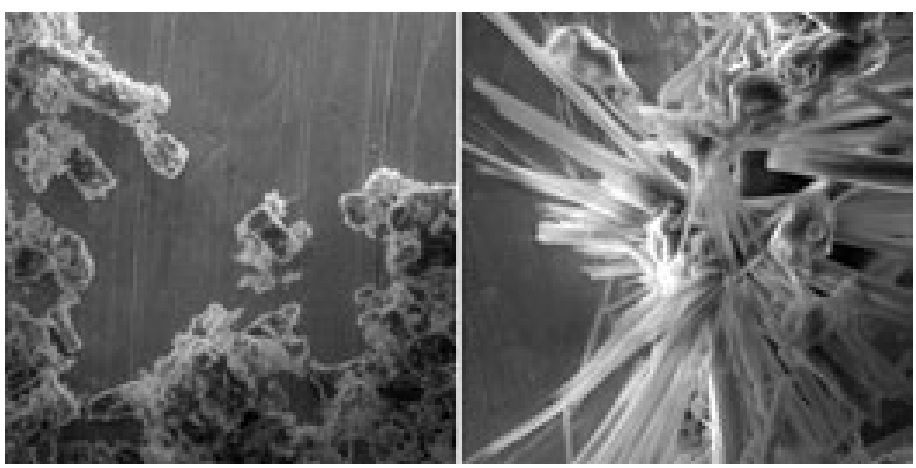

Figure 8 Dramatic changes in the crystal morphology before and after a hydration and dehydration cycle - left before hydration, right after dehydration.

of temperature and pressure capabilities, products can be observed through repeated temperature and humidity or wetting and drying cycles, allowing a in-situ simulation of accelerated shelf life tests (fig 6, 7, 8).

\section{Conclusion}

The use of SEM in pharmaceutical manufacturing is increasing rapidly. The growth is driven by changes in the regulatory environment intended to encourage well founded process improvements by changes in drug formulations and active pharmaceutical ingredients that are pushing resolution requirements beyond the capability of light microscopes, and by advances in microscope technology that have improved the usability and reduce the sample preparation burden of SEM. All of these trends are likely to continue and SEM will play an increasingly significant role in pharmaceutical manufacturing.

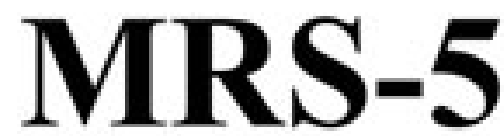

We are ISO-9000 certified and ISO-17025 accredited Microscopy Calibration Standard Now you can calibrate from $1,000 \times$ to $1,000,000 \mathrm{X}$ !

This is our fourth generation, traceable, magnification reference standard for all types (SEM, FESEM, Optical, STM. AFM, ete.) of microscopy. The MRS-5 has multiple $X$ and $Y$ pitch patterns ranging from $80 \mathrm{~nm}( \pm 1 \mathrm{~nm})$ to $2 \mu \mathrm{m}$ and 3 bar targets from $80 \mathrm{~nm}$ to $3 \mu \mathrm{m}$.

There is also a STM test pattem.

Free web resource guide!

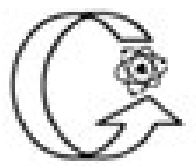

\section{GELLER} MICROÁNALYTICAL LABORATORY, Inc.

\author{
$426 \mathrm{e}$ Boston St., Topsfield, Ma 01983 \\ www.gellermicro.com
}

